\title{
Intraorbital Foreign Body With Intracranial Extension: A Case Series
}

\author{
Abhishek Singh*, Shahid Iftekhar Sadique, Samarendra Nath Ghosh \\ Department of Neurosurgery, Bangur Institute of Neurosciences, I.P.G.M.E.\&R., Kolkata, India
}

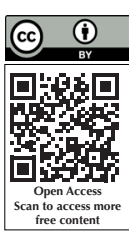

\begin{abstract}
Intraorbital foreign body with intracranial extension is a potentially devastating condition leading to blindness and even death in certain circumstances. Lot of controversies still exist regarding the most appropriate approach for removal of orbital foreign body with intracranial extension. In this paper we have discussed a series of 3 cases where the foreign body was removed through the anterior (transorbital) approach.
\end{abstract}

Keywords: Intraorbital; Intracranial; Foreign body; Extension

Citation: Singh A, Sadique SI, Ghosh SN. Intraorbital foreign body with intracranial extension: A case series. Int Clin Neurosci J. 2018;5(1):43-45. doi:10.15171/icnj.2018.08

\section{Introduction}

Even though orbital foreign body is a common complication of trauma, it is relatively rare in civilian life. Common causes for orbital foreign bodies are gunshot, missile and splinter injuries whereas in civilian population organic matter such as wooden bodies are common etiologic agents. ${ }^{1}$ Due to the peculiar anatomic characteristics of the orbit and periorbital area, orbital foreign bodies can extend into the intracranial cavity and can lead to significant morbidity and mortality if not treated adequately. Both, transcranial as well as anterior (transorbital) approach is mentioned in literature for removal of foreign bodies with intracranial extensions with each having its pros and cons.

\section{Case Presentation}

Case 1

A 34-year-old male patient presented to us in outpatient department with a wooden object into his left eye. This object gained access into his eye accidentally while he fell down from height at work. Patient was immediately admitted in neurosurgical ward and thorough history including mode of injury, detailed physical examinations including neurological and ophthalmological examinations were performed followed by CT scan of brain. Ophthalmological examination revealed complete loss of vision in affected eye whereas CT scan of brain revealed pneumocephalus and a small haemorrhagic contusion in left frontal lobe. Following CT scan we planned for surgical exploration and removal of foreign body and managed haemorrhagic contusion and pneumocephalus conservatively (Figure 1).

\section{Case 2}

A 16-year-old girl presented to us with a metallic foreign body in her left orbit. She belonged to a tribal community in West Bengal, and got accidentally hit by this foreign body (arrow) while she was sitting near window at her school. Patient's relatives tried to remove the arrow by pulling it from outside but as a result of this, the wooden part of the arrow got detatched from the intraorbital part and the metallic part remained within the orbit. We performed the same procedure of history, physical examination followed by CT brain including CT angiography. Ophthalmological examination revealed complete loss of vision. CT scan showed a foreign body (arrow) piercing the left orbit and gaining access into the right middle cranial fossa (Figure 2).

\section{Case 3}

A 9-year-old boy was brought by his parents with history of accidental entry of pencil into his left eye while playing with his sister. On examination the pencil end was not

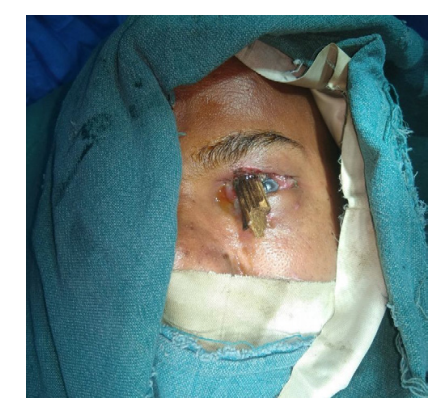

Figure 1. Wooden Foreign Body in Left Orbit Gaining Entrance Through Left Medial Canthus. 


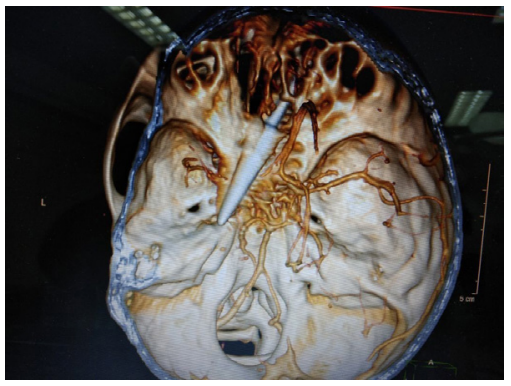

Figure 2. CT Angiography Brain Shows Foreign Body in Left Orbit Crossing Midline and Extending up to Right Middle Cranial Fossa.

visible from outside and the child had normal vision in both the eyes. We performed a CT scan brain with CT angiography and found a linear foreign body (graphite) gaining entrance into the left middle cranial fossa through the left orbit lateral to the left cavernous sinus (Figure 3).

\section{Treatment}

All these patients were given injection tetanus toxoid on admission and kept under antibiotic coverage. Seizure prophylaxis was started in all the patients. We planned for surgical exploration and removal of foreign bodies in presence of ophthalmologist. The major controversy here was to opt between the intracranial and the transorbital approach for removal of foreign body. However, based upon certain literature we proceeded with the transorbital approach. Under general anaesthesia we made an incision in the skin at the entry site of foreign body and traction sutures were applied to the skin to increase the exposure. Blunt dissection was carried along the tract of foreign body to separate it from the surrounding tissues. When we perceived that we have gained full access of the foreign body, it was pulled out with a gentle traction. Procedure was uneventful in all the 3 cases.

In postoperative period we continued with antibiotics and seizure prophylaxis and all the patients were discharged in a stable condition. On follow up 2 patients with history of vision loss following injury were still blind while the third patient had normal vision.

\section{Discussion}

Due to the conical shape of the orbit and thinness of orbital wall on medial side, any orbital foreign body can gain access into the intracranial cavity through superior orbital fissure or optic canal and can lead to blindness and death. ${ }^{2,3}$ If it gains access through superior orbital fissure, it is directed towards cavernous sinus, which is reflected in our study as well. ${ }^{4}$ Signs and symptoms depends upon the nature of foreign body, extent of penetration and injury to the neurovascular structures. ${ }^{5}$

Overall organic foreign bodies cause more inflammatory reactions, so, chances of infections and endophthalmitis are more and signs and symptoms are more obvious. ${ }^{6}$ $\mathrm{CT}$ scan can be considered the investigation of choice in

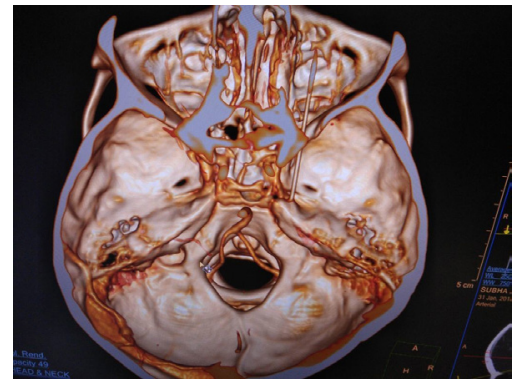

Figure 3. 3-D CT shows a linear foreign body (graphite within the pencil) extending from left orbit to left middle cranial fossa.

diagnosing and planning of management. ${ }^{7}$ Even though magnetic resonance imaging (MRI) may be helpful in certain circumstances but result may be devastating if nature of the foreign body is not known. As far as visual prognosis and status of neurovascular structures are concerned, it is related more to the direct impact from foreign body rather than surgical approaches. ${ }^{8}$ Intraorbital foreign body with intracranial extension can be removed via the transcranial as well as anterior (transorbital) approach. There is always a risk of bleeding and death following removal of foreign body from anterior approach due to sudden release of tamponading effect caused by foreign body over the lacerated vessels. Transcranial approach should be the procedure of choice if major neurovascular injury is suspected as it provides with better control to the neurovascular structures.

However, in recent years, imaging technologies such as CT Angiography with 3-D reconstruction have been particularly helpful to rule out vascular injury preoperatively. Transorbital approach can be considered a good alternative to the transcranial approach in situations where vascular injury is ruled out preoperatively as it is relatively simple and less morbid and patients do not have to go through the major trauma of craniotomy. Taking into account available literature and our own experience, we are of the view that if the patient is neurologically and haemodynamically stable after the injury and imaging studies rules out major neurovascular injury, anterior approach can be a safe and effective alternative for removal of intraorbital foreign bodies with intracranial extensions.

\section{Conclusion}

Intraorbital foreign body with intracranial extension can be managed via anterior (transorbital) approach safely if major neurovascular injury is ruled out.

Conflict of Interest Disclosures

The authors declare that they have no conflict of interests.

\section{Ethical Statement}

All patients gave informed consent before participating in this study.

\section{References}

1. Cho RI, Kahana A, Patel B, Sivak-Callcott J, Buerger DE, Durairaj $\mathrm{VD}$, et al. Intraoperative fluoroscopy-guided removal of orbital 
foreign bodies. Ophthal Plast Reconstr Surg. 2009;25(3):215-8. doi: 10.1097/IOP.0b013e3181a3006a.

2. Turbin RE, Maxwell DN, Langer PD, Frohman LP, Hubb B, Wolansky L, et al. Patterns of transorbital intracranial injury: a review and comparison of occult and non-occult cases. Surv Ophthalmol. 2006;51(5):449-60. doi: 10.1016/j. survophthal.2006.06.008.

3. Lasky JB, Epley KD, Karesh JW. Household objects as a cause of self-inflicted orbital apex syndrome. J Trauma. 1997;42(3):555-8.

4. Matsumoto S, Hasuo K, Mizushima A, Mihara F, Fukui M, Shirouzu T, et al. Intracranial penetrating injuries via the optic canal. AJNR Am I Neuroradiol. 1998;19(6):1163-5.

5. Santos Tde S, Melo AR, Moraes HH, Almeida Junior P, Dourado
E. Impacted foreign bodies in orbital region: review of nine cases. Arq Bras Oftalmol. 2010;73(5):438-42.

6. Potapov AA, Eropkin SV, Kornienko VN, Arutyunov NV, Yeolchiyan SA, Serova NK, et al. Late diagnosis and removal of a large wooden foreign body in the cranio-orbital region. J Craniofac Surg. 1996;7(4):311-4.

7. Wilson WB, Dreisbach JN, Lattin DE, Stears JC. Magnetic resonance imaging of nonmetallic orbital foreign bodies. Am J Ophthalmol. 1988;105(6):612-7.

8. Finkelstein M, Legmann A, Rubin PA. Projectile metallic foreign bodies in the orbit: a retrospective study of epidemiologic factors, management, and outcomes. Ophthalmology. 1997;104(1):96103. 